\title{
ERROR CALCULUS AND PATH SENSITIVITY IN FINANCIAL MODELS
}

\author{
Nicolas Bouleau \\ Ecole des Ponts, ParisTech
}

\begin{abstract}
In the framework of risk management, for the study of the sensitivity of pricing and hedging in stochastic financial models to changes of parameters and to perturbations of the stock prices, we propose an error calculus which is an extension of the Malliavin calculus based on Dirichlet forms. Although useful also in physics, this error calculus is well adapted to stochastic analysis and seems to be the best practicable in finance.

This tool is explained here intuitively and with some simple examples.

KEY WorDs: error calculus, risk management, Black-Scholes model, level dependent volatility, sensitivity, Greeks, Malliavin calculus, gradient, Ornstein-Uhlenbeck process, Dirichlet forms, Wiener space, carré du champ.
\end{abstract}

\section{INTRODUCTION}

Once a model is chosen to price contingent claims and to hedge a position, the actual questions obviously are : what is the exposure to errors on the model and to changes in the market? This risk assessment is usually done in terms of sensitivity of the portfolio to variations of numerical financial quantities and parameters of the model. Now the theory of Dirichlet forms allows to extend this sensitivity calculus to perturbations of functional quantities like stochastic processes. The method is an extension of Malliavin calculus. The errors are thought to be infinitesimal random quantities with biases, variances and covariances.

Among the promising clues of application of error calculus to finance, let us mention some directions : to manage the precision of numerical methods used to implement the stochastic theory; to obtain new integration by parts formulas to speed up Monte Carlo simulations ; to study how depends the solution of an ODE or of an SDE on a functional coefficient like the level-type volatility. We sketch these two last directions at the end of the article.

Our presentation starts from the basic ideas and goes until examples of completely tractable computations.

\section{PRESENTATION OF THE METHOD}

Several technics are available to represent errors mathematically and to compute them with formulas. The method of Dirichlet forms is based on some major historical ideas which are the easiest way to penetrate it. 


\subsection{Propagation of errors : error calculus à la Gauss}

After his argument showing the importance of the normal law (Theoria motus corporum coelestium 1809), Gauss was interested in the propagation of errors (Theoria Combinationis 1821). Given a quantity $U=F\left(V_{1}, V_{2}, \ldots\right)$ function of other erroneous quantities $V_{1}, V_{2}, \ldots$ he states the problem of computing the quadratic error to fear on $U$ knowing the quadratic errors $\sigma_{1}^{2}, \sigma_{2}^{2}, \ldots$ on $V_{1}, V_{2}, \ldots$, these errors being supposed small and independent. His answer is the following formula

$$
\sigma_{U}^{2}=\left(\frac{\partial F}{\partial V_{1}}\right)^{2} \sigma_{1}^{2}+\left(\frac{\partial F}{\partial V_{2}}\right)^{2} \sigma_{2}^{2}+\cdots
$$

he gives also the covariance between the error on $F$ and the error of an other function of the $V_{i}$ 's.

Formula (2.1) possesses a property which makes it highly better, in several questions, than other formulas used here and there in textbooks during the 19th and 20th centuries. It is a coherence property. By lack of place we refer to Bouleau (2001) $\S 1$ for the reason of this coherence property.

In the calculus à la Gauss the errors on $V_{1}, V_{2}, \ldots$ are not necessarily supposed to be independent nor constant, they can depend on $V_{1}, V_{2}, \ldots$ : Let be given a field of symmetric positive matrices $\left(\sigma_{i j}\left(v_{1}, v_{2}, \ldots\right)\right)$ on $\mathbb{R}^{d}$ representing the conditional variances and covariances of the errors on $V_{1}, V_{2}, \ldots$ given the values $v_{1}, v_{2}, \ldots$ of $V_{1}, V_{2}, \ldots$ then the error on $U=F\left(V_{1}, V_{2}, \ldots\right)$ is

$$
\sigma_{F}^{2}=\sum_{i j} \frac{\partial F}{\partial V_{i}}\left(v_{1}, v_{2}, \ldots\right) \frac{\partial F}{\partial V_{j}}\left(v_{1}, v_{2}, \ldots\right) \sigma_{i j}\left(v_{1}, v_{2}, \ldots\right)
$$

which depends solely on $F$ as mapping.

\subsection{First order and second order calculus}

The following remark, although very simple, is important to understand the role of the error calculus à la Gauss that will be used in the sequel in the extended form allowed by Dirichlet forms.

Let us start with a quantity $x$ with a small centred error $\varepsilon Y$, on which acts a non-linear regular function $f$. Thus we have at the beginning a random variable written $x+\varepsilon Y$, it has no bias (centred at the true value $x$ ) and its variance is $\varepsilon^{2} \sigma_{Y}^{2}$.

After having applied the function $f$, using Taylor formula shows that the error is no more centred. The bias has the same order of magnitude as the variance. Then applying new regular non-linear functions $f_{n}$ gives a transport formula which shows how errors propagate : biases and variances keep permanently the same order of magnitude

$$
\begin{aligned}
\text { bias }_{n+1} & =\operatorname{bias}_{n} f_{n+1}^{\prime}\left(x_{n}\right)+\frac{1}{2} \text { variance }_{n} f_{n+1}^{\prime \prime}\left(x_{n}\right)+\varepsilon^{3} 0(1) \\
\text { variance }_{n+1} & =\text { variance }_{n} f_{n+1}^{\prime 2}\left(x_{n}\right)+\varepsilon^{3} 0(1)
\end{aligned}
$$

(it could be easily extended to applications from $\mathbb{R}^{p}$ to $\mathbb{R}^{q}$, for the general formulas on the bias and the variance of the error under regular mappings see Bouleau and Hirsch (1991) chapter I $\S 6$ corollaries 6.1.3 and 6.1.4). 
We see that the calculus on the biases is a second order calculus involving the variance. Instead, the calculus on the variances is a first order calculus not involving the biases. Surprisingly, the calculus on the second order moments of errors is indeed simpler than the calculus on the first moments. Thus, the error calculus on the variances appears to be necessarily the first step in an analysis of errors propagation based on differential methods and supposing small errors.

\subsection{Extended error calculus using Dirichlet forms}

The error calculus of Gauss has the limitation that it has no mean of extension. If the error on $\left(V_{1}, V_{2}, V_{3}\right)$ is known it gives the error on any differentiable function of $\left(V_{1}, V_{2}, V_{3}\right)$ but that's all.

Now, in the usual probabilistic situations where a sequence of quantities $X_{1}, X_{2}, \ldots, X_{n}, \ldots$ is given and where the errors are known on the regular functions of a finite number of them, we would like to deduce the error on a function of an infinite number of the $X_{i}$ 's or at least on some such functions.

It is actually possible to reinforce this error calculus giving it a powerful extension tool and preserving the coherence property. In addition, it will give us the comfortable feature to handle Lipschitz functions as well.

For this we come back to the idea that the erroneous quantities are themselves random, as Gauss had supposed for his proof of the 'law of errors', say defined on $(\Omega, \mathcal{A}, \mathbb{P})$. The quadratic error on a random variable $X$ is then itself a random variable that we will denote by $\Gamma[X]$. Intuitively we still suppose the errors are infinitely small although this doesn't appear in the notation. It is as if we had an infinitely small unit to measure errors fixed in the whole problem. The extension tool is the following, we assume that if $X_{n} \rightarrow X$ in $L^{2}(\Omega, \mathcal{A}, \mathbb{P})$ and if the error $\Gamma\left[X_{m}-X_{n}\right]$ on $X_{m}-X_{n}$ can be made as small as we want in $L^{1}(\Omega, \mathcal{A}, \mathbb{P})$ for $m, n$ large enough, then the error $\Gamma\left[X_{n}-X\right]$ on $X_{n}-X$ goes to zero in $L^{1}$.

This can be axiomatized as follows : we call error structure a probability space equipped with a local Dirichlet form possessing a carré du champ.

Definition 2.1. An error structure is a term $(\Omega, \mathcal{A}, \mathbb{P}, \mathbb{D}, \Gamma)$ where $(\Omega, \mathcal{A}, \mathbb{P})$ is a probability space, satisfying the four properties :

1.) $\mathbb{D}$ is a dense subvectorspace of $L^{2}(\Omega, \mathcal{A}, \mathbb{P})$.

2.) $\Gamma$ is a positive symmetric bilinear map from $\mathbb{D} \times \mathbb{D}$ into $L^{1}(\mathbb{P})$ fulfilling the functional calculus of class $\mathcal{C}^{1} \cap$ Lip, what means that if $u \in \mathbb{D}^{m}$ and $v \in \mathbb{D}^{n}$ for $F$ and $G$ of class $\mathcal{C}^{1}$ and Lipschitz from $\mathbb{R}^{m}\left[\right.$ resp. $\left.\mathbb{R}^{n}\right]$ into $\mathbb{R}$, one has $F \circ u \in \mathbb{D}$ and $G \circ v \in \mathbb{D}$ and

$$
\Gamma[F \circ u, G \circ v]=\sum_{i, j} F_{i}^{\prime}(u) G_{j}^{\prime}(v) \Gamma\left[u_{i}, v_{j}\right] \quad \mathbb{P}-p . s . .
$$

3.) the bilinear form $\mathcal{E}[f, g]=\mathbb{E} \Gamma[f, g]$ is closed, i.e. $\mathbb{D}$ is complete under the norm $\|.\|_{\mathbb{D}}=\left(\|\cdot\|_{L^{2}(\mathbb{P})}^{2}+\mathcal{E}[., .]\right)^{\frac{1}{2}}$.

4.) $1 \in \mathbb{D}$ and $\Gamma[1,1]=0$.

We always write $\mathcal{E}[f]$ for $\mathcal{E}[f, f]$ and $\Gamma[f]$ for $\Gamma[f, f]$.

With this definition, the form $\mathcal{E}$ defined at point 3.) is a Dirichlet form. This notion has been introduced by A. Beurling and J. Deny as a tool in potential theory, see Beurling and Deny (1958-59), and also Fukushima, Oshima and Takeda (1994). 
The operator $\Gamma$ is the carré du champ or squared field operator associated with $\mathcal{E}$, it has been studied by several authors in more general contexts, see Dellacherie and Meyer (1987), Bouleau and Hirsch (1991). Here we refer to $\Gamma$ as the quadratic error operator of the error structure. Its intuitive meaning is the conditional variance of the error.

EXAMPlE 2.1. A simple example of error structure is the term

$$
\left(\mathbb{R}, \mathcal{B}(\mathbb{R}), m, H^{1}(m), \gamma\right)
$$

where $m$ is the normal law $N(0,1)$ and

$$
H^{1}(m)=\left\{f \in L^{2}(m): f^{\prime} \text { in the sense of distributions } \in L^{2}(m)\right\}
$$

with $\gamma[f]=f^{\prime 2}$ for $f \in H^{1}(m)$. This structure is associated to the real valued Ornstein-Uhlenbeck process. It models an erroneous quantity, say $X$, with normal law whose error does not depends on the value of $X$. Instead, the operator $\gamma[f](x)=f^{\prime 2}(x) x^{2}$ would model an error proportional to $X$, that is, in the sense of physicists, a constant proportional error. The intuitive relation giving the interpretation of the quadratic error operator $\gamma$ is

$$
\gamma[f](x)=\mathbb{E}\left[(\text { error on } f(X))^{2} \mid X=x\right] .
$$

\subsection{How proceeds an error calculation}

Let us suppose we are drawing a triangle with a graduated rule and a protractor: we take the polar angle of $O A$ say $\theta_{1}$, we put $O A=\ell_{1}$, then we take the angle $(O A, A B)$ say $\theta_{2}$, and we put $A B=\ell_{2}$.

1) Choose hypotheses on errors

$\ell_{1}, \ell_{2}$ and $\theta_{1}, \theta_{2}$ and their errors can be modeled by the following probability space and operator : :

$$
\left((0, L)^{2} \times(0, \pi)^{2}, \mathcal{B}\left((0, L)^{2} \times(0, \pi)^{2}\right), \frac{d \ell_{1}}{L} \frac{d \ell_{2}}{L} \frac{d \theta_{1}}{\pi} \frac{d \theta_{2}}{\pi}, \mathbb{D}, \Gamma\right)
$$

where $\mathbb{D}=\left\{f \in L^{2}\left(\frac{d \ell_{1}}{L} \frac{d \ell_{2}}{L} \frac{d \theta_{1}}{\pi} \frac{d \theta_{2}}{\pi}\right): \frac{\partial f}{\partial \ell_{1}}, \frac{\partial f}{\partial \ell_{2}}, \frac{\partial f}{\partial \theta_{1}}, \frac{\partial f}{\partial \theta_{2}} \in L^{2}\left(\frac{d \ell_{1}}{L} \frac{d \ell_{2}}{L} \frac{d \theta_{1}}{\pi} \frac{d \theta_{2}}{\pi}\right)\right\}$ and

$$
\Gamma[f]=\ell_{1}^{2}\left(\frac{\partial f}{\partial \ell_{1}}\right)^{2}+\ell_{1} \ell_{2} \frac{\partial f}{\partial \ell_{1}} \frac{\partial f}{\partial \ell_{2}}+\ell_{2}^{2}\left(\frac{\partial f}{\partial \ell_{2}}\right)^{2}+\left(\frac{\partial f}{\partial \theta_{1}}\right)^{2}+\frac{\partial f}{\partial \theta_{1}} \frac{\partial f}{\partial \theta_{2}}+\left(\frac{\partial f}{\partial \theta_{2}}\right)^{2},
$$

It is easily checked that assumptions 1) 2) 3) 4) of definition 2.1 are fulfilled.

2) Compute the errors on significant quantities using the functional calculus on $\Gamma$

For the coordinates of the point $B$ for example we have :

$$
\begin{aligned}
X_{B} & =\ell_{1} \cos \theta_{1}+\ell_{2} \cos \left(\theta_{1}+\theta_{2}\right) \quad Y_{B}=\ell_{1} \sin \theta_{1}+\ell_{2} \sin \left(\theta_{1}+\theta_{2}\right) \\
\Gamma\left[X_{B}\right] & =\ell_{1}^{2}+\ell_{1} \ell_{2}\left(\cos \theta_{2}+2 \sin \theta_{1} \sin \left(\theta_{1}+\theta_{2}\right)\right)+\ell_{2}^{2}\left(1+2 \sin ^{2}\left(\theta_{1}+\theta_{2}\right)\right) \\
\Gamma\left[Y_{B}\right] & =\ell_{1}^{2}+\ell_{1} \ell_{2}\left(\cos \theta_{2}+2 \cos \theta_{1} \cos \left(\theta_{1}+\theta_{2}\right)\right)+\ell_{2}^{2}\left(1+2 \cos ^{2}\left(\theta_{1}+\theta_{2}\right)\right) \\
\Gamma\left[X_{B}, Y_{B}\right] & =-\ell_{1} \ell_{2} \sin \left(2 \theta_{1}+\theta_{2}\right)-\ell_{2}^{2} \sin \left(2 \theta_{1}+2 \theta_{2}\right)
\end{aligned}
$$

Then, according to the problem, we can for example compute the covariance of the errors on the area and on the perimeter of the triangle, etc., or obtain that the proportional error on the area $\Gamma[$ area $] /$ area $^{2}$ is maximal for $\theta_{2}=\pi / 2$, etc. 
REMARK 2.1. If we limit our investigation to variances of the errors, that is to computation with $\Gamma$, then the choice of the a priori laws is not so crucial as it could be thought because these computations are done almost surely (using property 2 of definition 2.1. If we are, instead, interested also in biases, then the a priori laws are precisely relevant. Biases are represented by an operator which is the generator of the semi-group canonically associated with the error structure (see Bouleau and Hirsch (1991) chapter I). In example 2.1 it is $A[u](x)=\frac{1}{2} u^{\prime \prime}(x)-\frac{1}{2} x u^{\prime}(x)$. If we change the probability measure $m$ into $f . m$ ( $f$ regular), $\gamma$ being unchanged, then the operator $A$ becomes $\tilde{A}[u]=A[u]+\frac{1}{2 f} \gamma[f, u]$. The operator $u \rightarrow \frac{1}{2 f} \gamma[f, u]$ is first order. Absolutely continuous changes of the probability measure $m$ correspond to changes of the drift of the bias operator $A$, a variant of Girsanov theorem.

\subsection{Comparison of approaches}

Before looking at the infinite dimensional examples needed in finance, let us try to give an outlook over the different approaches to error calculus.

At the extreme right-hand side of the table we have the usual probability calculus in which the errors are random variables. The knowledge of the joint laws of the quantities and their errors is supposed to be yielded by statistical methods. The errors are finite, the propagation of the errors needs computation of image probability laws.

\begin{tabular}{|c|c|c|}
\hline deterministic & \multicolumn{2}{|c|}{ probabilistic approaches } \\
\hline $\begin{array}{l}\text { Sensitivity } \\
\text { calculus: } \\
\text { derivation with } \\
\text { respect to the } \\
\text { parameters of } \\
\text { the model }\end{array}$ & 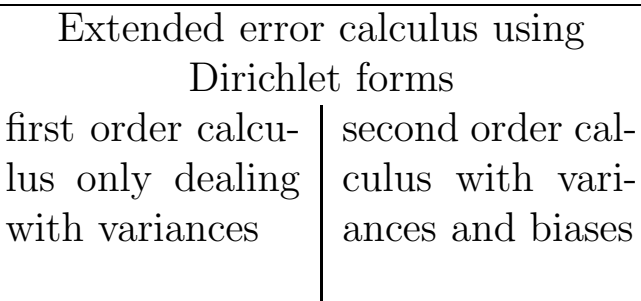 & $\begin{array}{c}\text { Probability } \\
\text { theory }\end{array}$ \\
\hline \multicolumn{2}{|r|}{ infinitesimal errors } & finite errors \\
\hline
\end{tabular}

Table 2.1: Main classes of error calculi

At the extreme left-hand side the usual sensitivity calculus consists of computing derivatives with respect to parameters. Let us remark that it applies also to functional coefficients using Fréchet or Gâteaux derivatives.

Between these two purely probabilistic and purely deterministic approaches lies the extended error calculus based on Dirichlet forms. It supposes the errors infinitely small but takes in account some features of the probabilistic approach allowing to put the computations and the arguments inside a powerful mathematical theory: the theory of Dirichlet forms. In the same framework can be performed either a first order calculus on variances which is simple and significant enough for most applications or a second order calculus dealing with both variances and biases.

\subsection{Main features of the method}

As above in the finite dimensional case of the triangle, the construction of an error structure on an infinite dimensional stochastic model is done in two steps 
1) If there are, as usually, deterministic parameters which can be erroneous or with respect to which a sensitivity is wished, these parameters have to be randomized with a priori laws.

2) Errors operators must be chosen to act on random quantities (initially random or randomized parameters) in order to describe errors, in such a way that we obtain mathematically an error structure.

Several properties of error structures make it easier such a construction.

1) The operation of taking the image of an error structure by a mapping is quite natural and gives an error structure as soon as the mapping, even non injective, satisfies some rather weak conditions. In particular if $(\Omega, \mathcal{A}, \mathbb{P}, \mathbb{D}, \Gamma)$ is an error structure and if $X$ is a random variable with values in $\mathbb{R}^{d}$ whose components are in $\mathbb{D},\left(\mathbb{R}^{d}, \mathcal{B}\left(\mathbb{R}^{d}\right), \mathbb{P}_{X}, \mathbb{D}_{X}, \Gamma_{X}\right)$ is an error structure where $\mathbb{P}_{X}$ is the law of $X$ and

$$
\begin{aligned}
\mathbb{D}_{X} & =\left\{f \in L^{2}\left(\mathbb{P}_{X}\right): f \circ X \in \mathbb{D}\right\} \\
\Gamma_{X}[f] & =\mathbb{E}[\Gamma[f \circ X] \mid X=x], \quad f \in \mathbb{D} .
\end{aligned}
$$

2) If $f \in \mathbb{D}$ and $F$ is Lipschitz from $\mathbb{R}$ to $\mathbb{R}$ then $F \circ f \in \mathbb{D}$ and $\Gamma[F \circ f] \leq k \Gamma[f]$. For example the structure of example $1\left(\mathbb{R}, \mathcal{B}(\mathbb{R}), m, H^{1}(m), \gamma\right)$ possesses an image by the map $x \rightarrow|\sin \sqrt{1+|x|}|$ which is an error structure on $[0,1]$. Such a use of non injective functions is tricky in the deterministic sensitivity calculus. More generally if $F$ is a contraction from $\mathbb{R}^{d}$ into $\mathbb{R}$ in the following sense

$$
|F(x)-F(y)| \leq \sum_{i=1}^{d}\left|x_{i}-y_{i}\right|
$$

then for $f_{1}, f_{2}, \ldots, f_{d} \in \mathbb{D}$ one has $F\left(f_{1}, f_{2}, \ldots, f_{d}\right) \in \mathbb{D}$ and

$$
\Gamma\left[F\left(f_{1}, f_{2}, \ldots, f_{d}\right)\right]^{\frac{1}{2}} \leq \sum_{i=1}^{d} \Gamma\left[f_{i}\right]^{\frac{1}{2}} .
$$

This property allows to consider more general images with values in metric spaces as soon as a suitable density property is preserved, see Bouleau-Hirsch (1991) chapter V $\S 1.3$ p 197.

3) The product of two or countably many error structures is an error structure. It is the mathematical expression of the independence of the random variables and the non-correlation of the errors. By this way error structures on infinite dimensional spaces are easily obtained, e.g. on the Wiener space, as we will see in the next part, or on the general Poisson space or other spaces of stochastic processes, see Bouleau and Hirsch (1991), Ma and Roeckner (1992), Bouleau (1995).

For later reference we give the following statement.

Theorem 2.1. Product structures

Let $S_{n}=\left(\Omega_{n}, \mathcal{F}_{n}, m_{n}, \mathbb{D}_{n}, \Gamma_{n}\right), n \geq 0$ be error structures.

The term $S=(\Omega, \mathcal{F}, m, \mathbb{D}, \Gamma)$ defined below is an error structure denoted $S=$ $\prod_{n=1}^{\infty} S_{n}$ and called the product structure of the $S_{n}$ :

$$
\begin{aligned}
& (\Omega, \mathcal{F}, m)=\left(\prod_{n=0}^{\infty} \Omega_{n}, \bigotimes_{n=0}^{\infty} \mathcal{F}_{n}, \prod_{n=0}^{\infty} m_{n}\right) \\
& \mathbb{D}=\left\{f \in L^{2}(m): \forall n, \text { for } m \text {-a.e. } \omega=\left(\omega_{0}, \omega_{1}, \ldots\right)\right. \\
& \text { the function } x \rightarrow f\left(\omega_{0}, \ldots, \omega_{n-1}, x, \omega_{n+1}, \ldots\right) \in \mathbb{D}_{n} \\
& \text { and } \left.\int \sum_{n} \Gamma_{n}[f] d m<+\infty\right\}
\end{aligned}
$$

and for $f \in \mathbb{D} \quad \Gamma[f]=\sum_{n} \Gamma_{n}[f]$. 
Thanks to these properties, is possible the construction of a variety of error structures on a given probabilistic model. Now for a rational treatment of a practical case these error hypotheses should be obtained by statistical methods. This is connected with the Fisher information theory, see Bouleau (2001). Anyhow, these statistical methods are not yet sufficiently studied to be exposed here, especially in the infinite dimensional case we have to use in finance. Thus we limit ourselves to error computations with a priori errors chosen the most likely we can. We will see that it is significant already.

\section{ERROR STRUCTURES ON THE WIENER SPACE}

Let us first recall the classical construction of the Brownian motion using the Wiener integral.

\subsection{The Wiener space as Gaussian product space}

Since we aim here at applications we will consider only the case where a measured space $(E, \mathcal{E}, \mu)$ is given which is either $\left(\mathbb{R}_{+}, \mathcal{B}\left(\mathbb{R}_{+}\right), d t\right)$ or $([0,1], \mathcal{B}([0,1]), d t)$ and a one-dimensional Brownian motion (for the abstract Wiener space setting see Bouleau and Hirsch (1991)).

Let $\left(\chi_{n}\right)$ be an orthonormal basis of $L^{2}(E, \mathcal{E}, \mu)$ and let $\left(g_{n}\right)$ be a sequence of i.i.d. reduced Gaussian variables defined on a probability space $(\Omega, \mathcal{A}, \mathbb{P})$. To each $f \in L^{2}(E, \mathcal{E}, \mu)$ we associate $I(f) \in L^{2}(\Omega, \mathcal{A}, \mathbb{P})$ by

$$
I(f)=\sum_{n}<f, \chi_{n}>g_{n}
$$

then $I$ is an isometric homomorphism from the Hilbert space $L^{2}(E, \mathcal{E}, \mu)$ into the Hilbert space $L^{2}(\Omega, \mathcal{A}, \mathbb{P})$. If $f$ and $g$ are orthogonal in $L^{2}(E, \mathcal{E}, \mu), I(f)$ and $I(g)$ are independent Gaussian random variables and putting

$$
B_{t}=\sum_{n}<1_{[0, t]}, \chi_{n}>g_{n} \quad\left(t \in[0,1] \text { or } t \in \mathbb{R}_{+}\right)
$$

defines a Gaussian stochastic process which is easily shown to be a standard Brownian motion. By extending the case where $f$ is a step function, the random variable $I(f)$ is denoted by $\int f(s) d B_{s}$ and defines the Wiener integral of $f$. In this construction we can suppose the space $(\Omega, \mathcal{A}, \mathbb{P})$ be a product space:

$$
(\Omega, \mathcal{A}, \mathbb{P})=(\mathbb{R}, \mathcal{B}(\mathbb{R}), m)^{\mathbf{N}} \quad m=N(0,1)
$$

and the $g_{n}$ 's be the coordinate mappings. Thus $\omega=\left(\omega_{0}, \ldots, \omega_{n}, \ldots\right)$ and $g_{n}(\omega)=$ $\omega_{n}$.

By the functional calculus, as soon as $\mathbb{D}$ and $\Gamma$ define an error structure on $(\Omega, \mathcal{A}, \mathbb{P})$, say $(\Omega, \mathcal{A}, \mathbb{P}, \mathbb{D}, \Gamma)$ for which the $g_{n}$ 's are in $\mathbb{D}$, this structure is determined by the quantities

$$
\Gamma\left[\int f(s) d B_{s}\right] \quad f \in D_{0} \text { dense in } L^{2}[0,1]\left(\text { resp. } L^{2}\left[\mathbb{R}_{+}\right]\right)
$$

because it follows that if $F \in \mathcal{C}^{1} \cap \operatorname{Lip}\left(\mathbb{R}^{k}\right)$

$$
\begin{aligned}
& \Gamma\left[F\left(\int f_{1} d B, \ldots, \int f_{k} d B\right)\right]= \\
& \sum_{i, j=1}^{k} F_{i}^{\prime}\left(\int f_{1} d B, \ldots\right) \quad F_{j}^{\prime}\left(\int f_{1} d B, \ldots\right) \Gamma\left[\int f_{i} d B, \int f_{j} d B\right]
\end{aligned}
$$


and the random variables $F\left(\int f_{1} d B, \ldots, \int f_{k} d B\right)$ for $F \in \mathcal{C}^{1} \cap \operatorname{Lip}\left(\mathbb{R}^{k}\right)$ and $f_{i} \in D_{0}$ are a dense subspace of $L^{2}(\mathbb{P})$.

\subsection{The Ornstein-Uhlenbeck structure}

Taking $\Gamma\left[\int f(s) d B_{s}\right]=\|f\|_{L^{2}}^{2}$ and $D_{0}=L^{2}[0,1]$ gives a closable structure which is of the form

$$
(\Omega, \mathcal{A}, \mathbb{P}, \mathbb{D}, \Gamma)=\prod_{n=0}^{\infty}\left(\left(\mathbb{R}, \mathcal{B}(\mathbb{R}), m, \mathbf{d}_{n}, \gamma_{n}\right)\right.
$$

where each factor $\left(\mathbb{R}, \mathcal{B}(\mathbb{R}), m, \mathbf{d}_{n}, \gamma_{n}\right)$ is here a copy of $\left(\mathbb{R}, \mathcal{B}(\mathbb{R}), m, H^{1}(m), \gamma\right)$ the Ornstein-Uhlenbeck structure of example 2.1. This error structure is induced by the following perturbation of the Brownian path :

$$
\omega(t) \longrightarrow \sqrt{e^{-\theta}} \omega(t)+\sqrt{1-e^{-\theta}} \hat{\omega}(t)
$$

where $\hat{\omega}$ is an independent Brownian motion and $\theta$ a vanishing parameter.

\subsection{Finer structures of product type}

Taking $\Gamma\left[\int f(s) d B_{s}\right]=\left\|f^{\prime}\right\|_{L^{2}}^{2}$ and $D_{0}=\mathcal{C}^{1}[0,1]$ or more generally for $q \in \mathbb{N}^{*}$ (or even $q \in \mathbb{R}_{+}^{*}$ ) $\Gamma\left[\int f(s) d B_{s}\right]=\left\|f^{(q)}\right\|_{L^{2}}^{2}$ and $D_{0}=\mathcal{C}^{q}[0,1]$ give also closable structures. For a suitable choice of the basis $\left(\chi_{n}\right)$ they are also of the form

$$
(\Omega, \mathcal{A}, \mathbb{P}, \mathbb{D}, \Gamma)=\prod_{n=0}^{\infty}\left(\left(\mathbb{R}, \mathcal{B}(\mathbb{R}), m, \mathbf{d}_{n}, \gamma_{n}\right)\right.
$$

\subsection{Error structures of generalised Mehler type}

Let $p_{t}$ be a strongly continuous symmetric contraction semi-group on $L^{2}\left(\mathbb{R}_{+}, d t\right)$ with generator $(a, \mathcal{D} a)$ ( $a$ being a non necessarily local operator), let us consider the associated closed positive quadratic form $(\varepsilon, \mathcal{D}(\sqrt{-a}))$ defined by $\varepsilon[f]=\|\sqrt{-a} f\|^{2}$ (a non necessarily Dirichlet form), then the structure on the Wiener space induced by the formula

$$
\Gamma\left[\int_{0}^{\infty} f(s) d B_{s}\right]=\varepsilon[f], \quad f \in \mathcal{D}(\sqrt{(-a)})
$$

is closable and thus defines an error structure.

It corresponds to the semigroup $P_{t}$ on $L^{2}(\Omega, \mathcal{A}, \mathbb{P})$ given by

$$
P_{t} F=\tilde{\mathbb{E}}\left[F\left(\int_{0}^{\infty}\left(p_{t / 2} 1_{[0, .]}\right)(u) d B_{u}+\int_{0}^{\infty}\left(\sqrt{I-p_{t}} 1_{[0, .]}\right)(v) d \tilde{B}_{v}\right)\right]
$$

where $\sqrt{I-p_{t}}$ is the positive square root of the positive operator $I-p_{t}$ on $L^{2}\left(\mathbb{R}_{+}, d t\right)$ and $\tilde{B}$ is an auxiliary independent Brownian motion.

REMARK 3.1. When a financial model is studied by means of a development in series with respect to a small random change in the coefficients (like small noise expansion of a stochastic volatility) it is possible to induce from the perturbation an error structure which manages the variances and the biases at the limit when the perturbation is infinitely small. We cannot describe the details here of this standard method. That yields often error structures outside the class of generalized Mehler type, but still defined by the quantities (3.2). 


\subsection{The gradient operator and the derivative}

In any error structure whose space $\mathbb{D}$ is separable, we can define a gradient operator $D$ on $\mathbb{D}$ with values in $L^{2}(\mathbb{P}, H)$ where $H$ is an auxiliary Hilbert space: $D$ is a continuous application from $\mathbb{D}$ into $L^{2}(\mathbb{P}, H)$ such that

1) $\forall U, V \in \mathbb{D} \quad<D U, D V>_{H}=\Gamma[U, V]$

2) $\forall F \in \mathcal{C}^{1} \cap \operatorname{Lip}\left(\mathbb{R}^{d}\right), \forall X \in \mathbb{D} \quad D(F \circ X)=\sum_{i=1}^{d} F_{i}^{\prime} \circ X . D X_{i} \quad \mathbb{P}$-a.s. Then if $U, V \in \mathbb{D} \cap L^{\infty}$ which is an algebra, it holds $D(U V)=D U . V+U . D V$. For example in the case of the Ornstein-Uhlenbeck structure, taking $H=L^{2}\left(\mathbb{R}_{+}\right)$ gives

. $\forall h \in L^{2}\left(\mathbb{R}_{+}\right) \quad D_{\text {ou }}\left[\int h(s) d B_{s}\right]=h$

. with suitable hypotheses on the adapted processe $H_{t}$ (see Nualart (1995))

$$
D_{\text {ou }}\left[\int H_{s} d B_{s}\right](t)=H_{t}+\int\left(D_{o u} H_{s}\right)(t) d B_{s}
$$

. If $U \in \mathbb{D}_{\text {ou }}$ the Clark formula

$$
U=\mathbb{E} U+\int_{0}^{\infty} \mathbb{E}\left[D_{\text {оu }} U(t) \mid \mathcal{F}_{t}\right] d B_{t} .
$$

Now a slight variant of the gradient operator, the notion of 'derivative', is useful when computing errors on solutions of stochastic differential equations thanks to the tool of Ito's formula (this notion has been used and studied by Feyel and la Pradelle (1989)).

DEFinition 3.1. Let $\left(\hat{B}_{t}\right)_{t \geq 0}$ be an auxiliary independent Brownian motion. For $U \in \mathbb{D}$ the derivative $U^{\#}$ is a random variable depending on $\omega$ and $\hat{\omega}$ defined by

$$
U^{\#}=\int_{0}^{\infty}\left(D_{\text {оu }} U\right)(\omega, t) d \hat{B}_{t} .
$$

From the properties of the gradient one gets

. $\Gamma[U]=\hat{\mathbb{E}}\left[U^{\# 2}\right]$

. For $F \in \mathcal{C}^{1} \cap \operatorname{Lip} \quad(F \circ U)^{\#}=F^{\prime} \circ U . U^{\#}$

$\left(\int H_{s} d B_{s}\right)^{\#}=\int H_{s} d \hat{B}_{s}+\int H_{s}^{\#} d B_{s}$.

\subsection{The weighted Ornstein-Uhlenbeck case}

Its meaning for financial models is to consider non necessarly time translation invariant perturbations of the underlying stock price. It is a special case of the generalised Mehler type:

$$
\begin{aligned}
& \quad \Gamma\left[\int f(t) d B_{t}\right]=\int \alpha(t) f^{2}(t) d t \quad f \in \mathcal{D}\left(\mathbb{R}_{+}\right) \quad \alpha \text { measurable } \geq 0 \\
& \text {. } \forall h \in L^{2}\left(\mathbb{R}_{+},(1+\alpha) d t\right) \quad D\left[\int h(s) d B_{s}\right]=\sqrt{\alpha(t)} h \\
& \text {. }\left(\int h(s) d B_{s}\right)^{\#}=\int \sqrt{\alpha(t)} h(t) d \hat{B}_{t} \\
& \text {. with suitable hypotheses on the adapted processe } H_{t} \\
& \quad D\left[\int H_{s} d B_{s}\right](t)=\sqrt{\alpha(t)} H_{t}+\int\left(D H_{s}\right)(t) d B_{s} \\
& \quad\left(\int H_{s} d B_{s}\right)^{\#}=\int H_{s} \sqrt{\alpha(s)} d \hat{B}_{s}+\int H_{s}^{\#} d B_{s} \\
& \text {. If } U \in \mathbb{D} \cap \mathbb{D}_{\text {ou }} \quad D U=\sqrt{\alpha} D_{o u} U
\end{aligned}
$$

The generator $(A, \mathcal{D} A)$ of this structure can easily be seen to verify

$$
A\left[\int f d B\right]=-\int \alpha(s) f(s) d B_{s} \quad f \in \mathcal{D}\left(\mathbb{R}_{+}\right)
$$

which permits (see formula (6.1) in the concluding remarks) to compute $A$ on a dense part of $\mathcal{D} A$. 
In the sequel, we focuse on the Ornstein-Uhlenbeck case, but the two following lemmas are also valid in the weighted Ornstein-Uhlenbeck case, and part III and IV extend to that case with only minor changes.

LEMMA 3.1. The conditional expectation operators $\mathbb{E}\left[\cdot \mid \mathcal{F}_{t}\right]$ are orthogonal projectors in $\mathbb{D}$ on errors sub-structures (closed sub-vector-spaces of $\mathbb{D}$ stable by Lipschitz functions).

LEMmA 3.2. Under the same hypotheses, let $\Gamma_{t}$ be defined from $\Gamma$ by

$$
\Gamma_{t}\left[\left(\int f(s) d B_{s}\right)\right]=\Gamma\left[\left(\int 1_{[0, t]} f(s) d B_{s}\right)\right]
$$

and let $U \rightarrow U^{\# t}$ the derivation operator associated with $\Gamma_{t}$, then for $U \in \mathbb{D}$ :

$$
\left(\mathbb{E}\left[U \mid \mathcal{F}_{t}\right]\right)^{\#}=\mathbb{E}\left[U^{\#} \mid \mathcal{F}_{t}\right]
$$

\section{APPLICATION TO FINANCIAL MODELS}

\subsection{The Black-Scholes case}

\section{Notation}

The interest rate for the bond is constant, the asset $\left(S_{t}\right)_{t \geq 0}$ is modeled as the solution of the equation $d S_{t}=S_{t}\left(\mu d t+\sigma d B_{t}\right)$. For a European option of the form $f\left(S_{T}\right), T$ fixed deterministic time (see Lamberton and Lapeyre (1997)), the value at time $t \in[0, T]$ of the option is $V_{t}=F\left(t, S_{t}, \sigma, r\right)$ with

$$
F(t, x, \sigma, r)=e^{-r(T-t)} \int_{\mathbb{R}} f\left(x e^{\left(r-\frac{\sigma^{2}}{2}\right)(T-t)+\sigma y \sqrt{T-t}}\right) \frac{e^{-\frac{y^{2}}{2}}}{\sqrt{2 \pi}} d y .
$$

If $f$ is Borel with linear growth, the function $F$ is $\mathcal{C}^{1}$ in $t \in\left[0, T\left[, \mathcal{C}^{2}\right.\right.$ and Lipschitz in $x \in] 0, \infty[$, let us put

$$
\text { delta }_{t}=\frac{\partial F}{\partial x}\left(t, S_{t}, \sigma, r\right) \quad \text { gamma }_{t}=\frac{\partial^{2} F}{\partial x^{2}}\left(t, S_{t}, \sigma, r\right)
$$

$F$ satisfies the equation $\frac{\partial F}{\partial t}+\frac{\sigma^{2} x^{2}}{2} \frac{\partial^{2} F}{\partial x^{2}}+r x \frac{\partial F}{\partial x}-r F=0$.

\section{Hypotheses}

Our choice is governed by an aim of simplicity.

a) The error on $\left(B_{t}\right)_{t \geq 0}$ is represented by the Ornstein-Uhlenbeck error structure.

b) The errors on the initial value $S_{0}$, on the volatility $\sigma$, on the rate $r$ are 'constant proportional errors' in the sense of physicists :

$$
\begin{aligned}
\Gamma\left[\phi\left(S_{0}\right)\right] & =\phi^{\prime 2}\left(S_{0}\right) S_{0}^{2} \\
\Gamma[\psi(\sigma)] & =\psi^{\prime 2}(\sigma) \sigma^{2} \\
\Gamma[\xi(r)] & =\xi^{\prime 2}(r) r^{2}
\end{aligned}
$$

c) We chose a priori laws : lognormal laws on $S_{0}$ and $\sigma$, an exponential law on $r$.

d) We suppose $\left(B_{t}\right)_{t \geq 0}$ and the randomized quantities are independent and their errors uncorrelated. (In a more complete study, these independence and 
uncorrelation assumptions would have to be relaxed, in particular to express links between errors on the asset $\left(S_{t}\right)$ and on the volatility $\left.\sigma\right)$.

In other words, the error on a regular function $F\left(\left(B_{t}\right)_{t \geq 0}, S_{0}, \sigma, r\right)$ will be represented by the product error structure i.e.

$$
\Gamma\left[F\left(\left(B_{t}\right)_{t \geq 0}, S_{0}, \sigma, r\right)\right]=\Gamma_{\text {ou }}\left[F\left(., S_{0}, \sigma, r\right)\right]+F_{S_{0}}^{\prime 2} S_{0}^{2}+F_{\sigma}^{\prime 2} \sigma^{2}+F_{r}^{\prime 2} r^{2}
$$

where $\Gamma_{o u}$ is the Ornstein-Uhlenbeck quadratic error operator.

Actually, the theory tells us that hedging and pricing formulas do not involve the drift coefficient $\mu$. So we may take $\mu=r$, i.e. we work under the probability $\mathbb{P}$ such that $\tilde{S}_{t}=e^{-r t} S_{t}$, the discounted stock price, is a martingale. Since $S_{t}=$ $S_{0} e^{\sigma B_{t}+\left(r-\frac{\sigma^{2}}{2}\right) t}$ we have

$$
\Gamma\left[S_{t}\right]=S_{t}^{2}\left\{\sigma^{2} \int_{0}^{t} \alpha(s) d s+\left(B_{t}-\sigma t\right)^{2} \sigma^{2}+t^{2}\right\}
$$

4.2. Errors on the value and the hedge of a European option

Let us consider an option of the form $f\left(S_{T}\right)$ where $f$ is Lipschitz.

By the independence hypothesis, the errors on $B, S_{0}, \sigma, r$ can be managed separately. Let us denote $\Gamma_{B}, \Gamma_{0}, \Gamma_{\sigma}, \Gamma_{r}$ the corresponding quadratic operators.

a) Error on the value of the option

The value of the option is $V_{t}=F\left(t, S_{t}, \sigma, r\right)$ with $F$ given by (4.1)

a1) Error due to $B$

$B$ being present only in $S_{t}$, we have $\Gamma_{B}\left[V_{t}\right]=\left(\frac{\partial F}{\partial x}\left(t, S_{t}, \sigma, r\right)\right)^{2} \Gamma_{B}\left[S_{t}\right]$ so

$$
\begin{aligned}
\Gamma_{B}\left[V_{t}\right] & =\operatorname{delta}_{t}^{2} \Gamma_{B}\left[S_{t}\right] \\
\Gamma_{B}\left[V_{s}, V_{t}\right] & =\operatorname{delta}_{s} \operatorname{delta}_{t} \Gamma_{B}\left[S_{s}, S_{t}\right]
\end{aligned}
$$

with $\Gamma_{B}\left[S_{s}, S_{t}\right]=S_{s} S_{t} \sigma^{2}(s \wedge t)$.

Proposition 4.1. If $f$ is Lipschitz, $V_{t}$ is in $\mathbb{D}_{B}$ and when $t \uparrow T$

$$
\begin{gathered}
V_{t}=F\left(t, S_{t}, \sigma, r\right) \rightarrow f\left(S_{T}\right) \quad \text { in } \mathbb{D}_{B} \text { and } \mathbb{P}-\text { a.s. } \\
\Gamma_{B}\left[V_{t}\right]=\left(\text { delta }_{t}\right)^{2} \Gamma_{B}\left[S_{t}\right] \rightarrow f^{\prime 2}\left(S_{T}\right) \Gamma_{B}\left[S_{T}\right] \quad \text { in } L^{1} \text { and } \mathbb{P}-\text { a.s. }
\end{gathered}
$$

proof. Let us suppose first $f \in \mathcal{C}^{1} \cap$ Lip. By the relation

$$
V_{t}=\mathbb{E}\left[e^{-r(T-t)} f\left(S_{T}\right) \mid \mathcal{F}_{t}\right]
$$

it follows that $V_{t} \rightarrow f\left(S_{T}\right)$ in $L^{p} \quad 1 \leq p<\infty$ and a.s.

A computation that we shall do in a more general framework later, and that we do not repeat here, gives

$$
V_{t}^{\#}=e^{-r(T-t)} \mathbb{E}\left[f^{\prime}\left(S_{T}\right) S_{T} \mid \mathcal{F}_{t}\right] \sigma \hat{B}_{t}
$$

thus

$$
V_{t}^{\#} \rightarrow f^{\prime}\left(S_{T}\right) S_{T} \sigma \hat{B}_{T} \text { in } L^{2}\left(\mathbb{P}, L^{2}(\hat{\Omega}, \hat{\mathbb{P}})\right)
$$

and thanks $f\left(S_{T}\right)^{\#}=f^{\prime}\left(S_{T}\right) S_{T} \sigma \hat{B}_{T}$ we obtain

$$
V_{t} \rightarrow f\left(S_{T}\right) \quad \text { in } \mathbb{D}_{B} \text { and } \mathbb{P}-\text { a.s. }
$$


and

$$
\Gamma_{B}\left[V_{t}\right]=e^{-2 r(T-t)}\left(\mathbb{E}\left[f^{\prime}\left(S_{T}\right) S_{T} \mid \mathcal{F}_{t}\right]\right)^{2} \sigma^{2} t \rightarrow f^{\prime 2}\left(S_{T}\right) \Gamma_{B}\left[S_{T}\right]
$$

in $L^{1}$ and $\mathbb{P}$-a.s.

The case $f$ only Lipschitz comes from a special property of the one-dimentional functional calculus in error structures (see Bouleau and Hirsch (1991) chapter III prop. 2.1.5), the preceding argument still remains valid. a2) Error due to $\sigma$.

We suppose here $f \in \mathcal{C}^{1} \cap$ Lip. As $V_{t}=F\left(t, S_{t}, \sigma, r\right)$

$$
\Gamma_{\sigma}\left[V_{t}\right]=\left\{F _ { x } ^ { \prime } \left(\left(t, S_{t}, \sigma, r\right) \frac{\partial S_{t}}{\partial \sigma}+F_{\sigma}^{\prime}\left(\left(t, S_{t}, \sigma, r\right)\right\}^{2} \sigma^{2}\right.\right.
$$

and the computation can be done using the integral representation (4.1), puting

$$
\tilde{F}(t, x, \sigma)=e^{r(T-t)} F\left(t, x e^{-r(T-t)}, \sigma, r\right)
$$

and remarking that by (4.1) we have

$$
\frac{\partial \tilde{F}}{\partial t}+\frac{\sigma^{2} x^{2}}{2} \frac{\partial^{2} \tilde{F}}{\partial x^{2}}=0 \quad \frac{\partial \tilde{F}}{\partial \sigma}=-\frac{2(T-t)}{\sigma} \frac{\partial \tilde{F}}{\partial t} \quad \frac{\partial F}{\partial \sigma}=(T-t) \sigma x^{2} \frac{\partial^{2} F}{\partial x^{2}}
$$

and we obtain

$$
\begin{aligned}
\Gamma_{\sigma}\left[V_{t}\right] & =\left\{(T-t) \sigma S_{t}^{2} \text { gamma }_{t}+S_{t}\left(B_{t}-\sigma t\right) \text { delta }_{t}\right\}^{2} \sigma^{2} \\
& =\left\{\text { vega }_{t}+S_{t}\left(B_{t}-\sigma t\right) \text { delta }_{t}\right\}^{2} \sigma^{2}
\end{aligned}
$$

One gets immediately, for example, the well-known fact that for two European options of payoffs $f_{(1)}\left(S_{T}\right)$ and $f_{(2)}\left(S_{T}\right)$, an option with payoff $a_{1} f_{(1)}\left(S_{T}\right)+a_{2} f_{(2)}\left(S_{T}\right)$ would have a value $V_{0}$ at $t=0$ insensitive to $\sigma$, i.e. $\Gamma_{\sigma}\left[V_{0}\right]=0$, as soon as $a_{1}$ gamma $_{0}^{(1)}+a_{2}$ gamma $_{0}^{(2)}=0$.

a3) Error due to $r$.

We have similarly

$$
\Gamma_{r}\left[V_{t}\right]=\left\{F _ { x } ^ { \prime } \left(\left(t, S_{t}, \sigma, r\right) \frac{\partial S_{t}}{\partial r}+F_{r}^{\prime}\left(\left(t, S_{t}, \sigma, r\right)\right\}^{2} r^{2}\right.\right.
$$

thus

$$
\Gamma_{r}\left[V_{t}\right]=\left\{t S_{t} \text { delta }_{t}+\mathrm{rho}_{t}\right\}^{2} r^{2} .
$$

As a consequence, given several options of payoffs $f_{(i)}\left(S_{T}\right), i=1, \ldots, k$, the option of payoff $\sum_{i} a_{i} f_{(i)}\left(S_{T}\right)$ has a value at $t=0$ insensitive to both $\sigma$ and $r$ (i.e. $\Gamma_{\sigma}\left[V_{0}\right]=$ $\left.\Gamma_{r}\left[V_{0}\right]=0\right)$ if the vector $a=\left(a_{i}\right)$ is orthogonal to the two vectors $\left(\operatorname{gamma}_{0}^{(i)}\right)$ and $\left(\mathrm{rho}_{0}^{(i)}\right)$.

b) Error on the hedging portfolio

Here we limit ourselves to the error due to $\left(B_{t}\right)$. We suppose $f$ and $f^{\prime}$ in $\mathcal{C}^{1} \cap$ Lip. The hedging equation is

$$
e^{-r t} F\left(t, S_{t}, \sigma, r\right)=F\left(0, S_{0}, \sigma, r\right)+\int_{0}^{t} H_{s} d \tilde{S}_{s}
$$

where the adapted process $H_{t}$ is the quantity of stock in the portfolio :

$$
H_{t}=\operatorname{delta}_{t}=\frac{\partial F}{\partial x}\left(t, S_{t}, \sigma, r\right)=e^{-r(T-t)} \mathbb{E}\left[f^{\prime}\left(S_{T}\right) S_{T} \mid \mathcal{F}_{t}\right] \frac{1}{S_{t}}
$$


By the same method as for $V_{t}$ we obtain

$$
\begin{aligned}
\Gamma_{B}\left[H_{t}\right] & \left.=\text { (gamma }_{t}\right)^{2} \Gamma_{B}\left[S_{t}\right] \\
\Gamma_{B}\left[H_{s}, H_{t}\right] & =\text { gamma }_{s} \text { gamma }_{t} \Gamma_{B}\left[S_{s}, S_{t}\right]
\end{aligned}
$$

Proposition 4.2. If $f, f^{\prime} \in \mathcal{C}^{1} \cap$ Lip, then $H_{t} \in \mathbb{D}$ and as $t \uparrow T$

$$
\begin{aligned}
H_{t} & \rightarrow f^{\prime}\left(S_{T}\right) \quad \text { in } \mathbb{D}_{B} \text { and a.s. } \\
\Gamma_{B}\left[H_{t}\right] & \rightarrow f^{\prime \prime 2}\left(S_{T}\right) \Gamma_{B}\left[S_{T}\right] \quad \text { in } L^{1}(\mathbb{P}) \text { and a.s. }
\end{aligned}
$$

REMARK 4.1. These results show that the Greeks introduced by practioners have a direct sense as sensitivity of the value $V_{t}$ and of the hedging $H_{t}$ to perturbations. This is of course not surprising, the method makes more precise the correlations of errors. It gives also a tool to study the absolute continuity of joint laws as we explain now.

The preceding computations show easily that in the Black-Scholes model, if $U_{1}$ and $U_{2}$ are two random variables taken among the following quantities defined at a fixed instant $t: S_{t}, V_{t}\left(f_{1}\right), V_{t}\left(f_{2}\right), H_{t}\left(f_{1}\right), H_{t}\left(f_{2}\right)$, then the matrix $\Gamma\left[U_{i}, U_{j}\right]$ is singular: the errors on these quantities are linked. This comes from the fact that the law of e.g. the pair $\left(V_{t}\left(f_{1}\right), V_{t}\left(f_{2}\right)\right)$ is carried by the $\lambda$-parametrized curve:

$$
\begin{aligned}
& y=\exp -r(T-t) P_{T-t} f_{1}(\lambda) \\
& x=\exp -r(T-t) P_{T-t} f_{2}(\lambda)
\end{aligned}
$$

where $\left(P_{t}\right)$ is the transition semigroup of $\left(S_{t}\right)$. The same phenomenon happens in any more general Markovian model.

On the contrary the random quantities involving several different instants have generally non-linked errors. Thus for example if $U_{1}=S_{T}$ and $U_{2}=\int_{0}^{T} e^{-s} H_{s} S_{s} d s$ (discounted immobilization of the portfolio) the matrix $\Gamma\left[U_{i}, U_{j}\right]$ is a.s. regular as soon as $f$ is not constant, hence, by the absolute continuity criterion (Bouleau and Hirsch (1986) or Nualart (1995) thm 2.1.2) the law of the pair $\left(S_{T}, \int_{0}^{T} e^{-s} H_{s} S_{s} d s\right)$ possesses a density.

c) More general errors on $\left(B_{t}\right)$

The relations (4.2) (4.3) (4.4) (4.5) still hold in the weighted Ornstein-Uhlenbeck case, and also, with suitable hypotheses, if we consider more general error structures on the Wiener space. Let us consider, as mentioned above, a structure induced by a closed positive quadratic form $\varepsilon$ on $L^{2}\left(\mathbb{R}_{+}, d t\right)$ with

$$
\Gamma_{B}\left[\int f d B\right]=\varepsilon[f]
$$

for $f$ in the domain of $\varepsilon$ with, for example,

$$
\begin{array}{ll}
\text { i) } & \varepsilon[f]=\int_{0}^{\infty} \int_{0}^{\infty}(f(s)-f(t))^{2} \beta(s) \beta(t) d s d t \\
\text { ii) } & \varepsilon[f]=\int_{0}^{1}\left(f^{(q)}(s)\right)^{2} d s
\end{array}
$$

where $f^{(q)}$ is the fractional derivative of order $q$, then the formulas

$$
\begin{aligned}
\Gamma_{B}\left[V_{t}\right] & =\left(\text { delta }_{t}\right)^{2} \Gamma_{B}\left[S_{t}\right] \\
\Gamma_{B}\left[H_{t}\right] & =\left(\text { gamma }_{t}\right)^{2} \Gamma_{B}\left[S_{t}\right]
\end{aligned}
$$


remain valid as soon as $S_{t} \in \mathbb{D}$ i.e.

in case i) if $\beta \in L^{1}\left(\mathbb{R}_{+}, d t\right)$ and $\Gamma_{B}\left[S_{t}\right]=S_{t}^{2} \sigma^{2} 2 \int_{t}^{\infty} \beta(s) d s \int_{\infty}^{t} \beta(s) d s$.

in case ii) if $q \in\left(0, \frac{1}{2}\right)$ and $\Gamma_{B}\left[S_{t}\right]=S_{t}^{2} \sigma^{2} \sum_{n=1}^{\infty} \frac{4(1-\cos 2 \pi n t)}{(2 \pi n)^{2(1-q)}}$.

Now in the case

$$
\text { iii) } \quad \varepsilon[f]=\int_{0}^{\infty}\left(\sum_{i=1}^{d} a_{i}(s) f^{(i)}(s)\right)^{2} d s
$$

we do not have anymore $1_{[0, t]} \in \operatorname{dom}(\varepsilon)$, hence $B_{t}$ doesn't belong to $\mathbb{D}$. Such error structures are more convenient to model errors on processes with finite variation.

\section{MODELS WITH LEVEL DEPENDENT VOLATILITY}

We will display the method in the case of a complete market, the probability being a martingale measure and for a simple one-dimensional diffusion model.

The stock is supposed to be the solution of the equation

$$
d X_{t}=X_{t} \sigma\left(t, X_{t}\right) d B_{t}+X_{t} r(t) d t
$$

We limit the study to the error due to $\left(B_{t}\right)$ which is defined by an Ornstein-Uhlenbeck structure:

$$
\Gamma\left[\int_{0}^{\infty} h(s) d B_{s}\right]=\int_{0}^{\infty} h^{2}(s) d s .
$$

The rate is deterministic, the function $\sigma(t, x)$ will be supposed bounded with bounded derivative in $x$ uniformly for $t \in[0, T]$.

Let $f\left(X_{T}\right)$ be a European option. Its value at time $t$ is

$$
V_{t}=\mathbb{E}\left[\exp \left(-\int_{t}^{T} r(s) d s\right) f\left(X_{T}\right) \mid \mathcal{F}_{t}\right]
$$

the hedging portfolio is given by the adapted process $H_{t}$ which satisfies

$$
\tilde{V}_{t}=\exp \left(-\int_{0}^{t} r(s) d s\right) V_{t}=V_{0}+\int_{0}^{t} H_{s} d \tilde{X}_{s}
$$

where $\tilde{X}_{s}=\exp \left(-\int_{0}^{t} r(s) d s\right) X_{t}$.

We proceed as follows: from the equation

$$
X_{t}=X_{0}+\int_{0}^{t} X_{s} \sigma\left(s, X_{s}\right) d B_{s}+\int_{0}^{t} r(s) X_{s} d s
$$

we obtain

$$
X_{u}^{\#}=\int_{0}^{u}\left(\sigma\left(s, X_{s}\right)+X_{s} \sigma_{x}^{\prime}\left(s, X_{s}\right)\right) X_{s}^{\#} d B_{s}+\int_{0}^{u} X_{s} \sigma\left(s, X_{s}\right) d \hat{B}_{s}+\int_{0}^{u} r(s) X_{s}^{\#} d s
$$

this equation is solved by putting

$$
\begin{aligned}
K_{s} & =\sigma\left(s, X_{s}\right)+X_{s} \sigma_{x}^{\prime}\left(s, X_{s}\right) \\
M_{u} & =\exp \left\{\int_{0}^{u} K_{s} d B_{s}-\frac{1}{2} \int_{0}^{u} K_{s}^{2} d s+\int_{0}^{u} r(s) d s\right\}
\end{aligned}
$$

and remarking that

$$
X_{u}^{\#}=M_{u} \int_{0}^{u} \frac{X_{s} \sigma\left(s, X_{s}\right)}{M_{s}} d \hat{B}_{s} .
$$

a) Let us first suppose $f \in \mathcal{C}^{1} \cap$ Lip and let us define $Y=\exp \left(-\int_{t}^{T} r(s) d s\right) f\left(X_{T}\right)$. To compute $\left(\mathbb{E}\left[Y \mid \mathcal{F}_{t}\right]\right)^{\#}$ we apply the second lemma of section 3:

$$
Y^{\#_{t}}=\exp \left(-\int_{t}^{T} r(s) d s\right) f^{\prime}\left(X_{T}\right) X_{T}^{\#_{t}}
$$


and

$$
\begin{gathered}
\left(\mathbb{E}\left[Y \mid \mathcal{F}_{t}\right]\right)^{\#}=\exp \left(-\int_{t}^{T} r(s) d s\right) \mathbb{E}\left[f^{\prime}\left(X_{T}\right) X_{T}^{\# t} \mid \mathcal{F}_{t}\right] \\
=\exp \left(-\int_{t}^{T} r(s) d s\right) \mathbb{E}\left[f^{\prime}\left(X_{T}\right) M_{T} \mid \mathcal{F}_{t}\right] \int_{0}^{t} \frac{X_{s} \sigma\left(s, X_{s}\right)}{M_{s}} d \hat{B}_{s}
\end{gathered}
$$

and the second lemma gives

$$
\begin{aligned}
\Gamma\left[V_{t}\right] & =\Gamma\left[\mathbb{E}\left[Y \mid \mathcal{F}_{t}\right]\right] \\
& =\exp \left(-2 \int_{t}^{T} r(s) d s\right)\left(\mathbb{E}\left[f^{\prime}\left(X_{T}\right) M_{T} \mid \mathcal{F}_{t}\right]\right)^{2} \int_{0}^{t} \frac{X_{s}^{2} \sigma^{2}\left(s, X_{s}\right)}{M_{s}^{2}} d s
\end{aligned}
$$

this yields also the cross error of $V_{s}$ and $V_{t}$ which is usefull to compute errors on random variables such that $\int_{0}^{T} h(s) d V_{s}$ or $\int_{0}^{T} h(s) V_{s} d s$.

$$
\begin{array}{r}
\Gamma\left[V_{s}, V_{t}\right]=\exp \left(-\int_{s}^{T} r(s) d s-\int_{t}^{T} r(s) d s\right) \\
\mathbb{E}\left[f^{\prime}\left(X_{T}\right) M_{T} \mid \mathcal{F}_{s}\right] \mathbb{E}\left[f^{\prime}\left(X_{T}\right) M_{T} \mid \mathcal{F}_{t}\right] \\
\int_{0}^{s \wedge t} \frac{X_{u}^{2} \sigma^{2}\left(u, X_{u}\right)}{M_{u}^{2}} d u .
\end{array}
$$

With our hypotheses as $t \uparrow T$

$$
\Gamma\left[V_{t}\right] \rightarrow f^{\prime 2}\left(X_{T}\right) M_{T}^{2} \int_{0}^{T} \frac{X_{s}^{2} \sigma^{2}\left(s, X_{s}\right)}{M_{s}^{2}} d s=f^{\prime 2}\left(X_{T}\right) \Gamma\left[X_{T}\right]
$$

in $L^{1}(\mathbb{P})$ and a.s.

b) Now to deal with $H_{t}$, let us remark first that $H_{t}$ is easily obtained by the Clark formula. The formula 5.1 gives

$$
H_{t} \exp \left(-\int_{0}^{t} r(s) d s\right) X_{t} \sigma\left(X_{t}\right)=D_{a d}\left[\exp \left(-\int_{0}^{T} r(s) d s\right) f\left(X_{T}\right)\right]
$$

where $D_{a d}$ is the adapted O-U-gradient defined by

$$
D_{a d}[Z](t)=\mathbb{E}\left[D Z(t) \mid \mathcal{F}_{t}\right]
$$

Since

$$
D\left[\exp \left(-\int_{0}^{T} r(s) d s\right) f\left(X_{T}\right)\right]=\exp \left(-\int_{0}^{T} r(s) d s\right) f^{\prime}\left(X_{T}\right)\left(D X_{T}\right)(t)
$$

we have from the computation done for $V_{t}$

$$
D\left[\exp \left(-\int_{0}^{T} r(s) d s\right) f\left(X_{T}\right)\right]=\exp \left(-\int_{0}^{T} r(s) d s\right) \mathbb{E}\left[f^{\prime}\left(X_{T}\right) M_{T} \mid \mathcal{F}_{t}\right] \frac{X_{t} \sigma\left(t, X_{t}\right)}{M_{t}} .
$$

Thus

$$
H_{t}=\exp \left(-\int_{t}^{T} r(s) d s\right) \mathbb{E}\left[f^{\prime}\left(X_{T}\right) M_{T} \mid \mathcal{F}_{t}\right] \frac{1}{M_{t}} .
$$

Now supposing $f$ and $f^{\prime} \in \mathcal{C}^{1} \cap$ Lip we apply the same method as for obtaining $\Gamma\left[V_{t}\right]$ which leads to

$$
\begin{aligned}
\Gamma\left[H_{t}\right]=\exp \left(-2 \int_{t}^{T} r(s) d s\right) & \\
& \left(\mathbb{E}\left[\frac{M_{T}}{M_{t}}\left(f^{\prime \prime}\left(X_{T}\right) M_{T}+f^{\prime}\left(X_{T}\right) Z_{t}^{T} \mid \mathcal{F}_{t}\right]\right)^{2} \int_{0}^{t} \frac{X_{u}^{2} \sigma^{2}\left(u, X_{u}\right)}{M_{u}^{2}} d u\right.
\end{aligned}
$$

$$
\text { with } \begin{aligned}
Z_{t}^{T} & =\int_{t}^{T} L_{s} d B_{s}-\int_{t}^{T} K_{s} L_{s} M_{s} d s \\
\text { and } \quad K_{s} & =\sigma\left(s, X_{s}\right)+X_{s} \sigma_{x}^{\prime}\left(s, X_{s}\right) \\
L_{s} & =2 \sigma_{x}^{\prime}\left(s, X_{s}\right)+X_{s} \sigma_{x^{2}}^{\prime \prime}\left(s, X_{s}\right) .
\end{aligned}
$$


If we introduce the following notation which, in our present Markovian model, gives the probabilistic interpretation of the usual Greeks

$$
\begin{aligned}
\text { delta }_{t} & =H_{t}=\exp \left(-\int_{t}^{T} r(s) d s\right) \mathbb{E}\left[f^{\prime}\left(X_{T}\right) M_{T} \mid \mathcal{F}_{t}\right] \frac{1}{M_{t}} \\
\text { gamma }_{t} & =\exp \left(-\int_{t}^{T} r(s) d s\right) \mathbb{E}\left[\frac{M_{T}^{2}}{M_{t}^{2}}\left(f^{\prime \prime}\left(X_{T}\right)+\frac{M_{T}}{M_{t}^{2}} f^{\prime}\left(X_{T}\right) Z_{t}^{T} \mid \mathcal{F}_{t}\right]\right.
\end{aligned}
$$

we can summarize some formulas of this case with level dependent volatility by

$$
\begin{aligned}
V_{t}^{\#} & =\operatorname{delta}_{t} X_{t}^{\#} & & \\
\Gamma\left[V_{t}\right] & =\operatorname{delta}_{t}^{2} \Gamma\left[X_{t}\right] & \Gamma\left[V_{s}, V_{t}\right] & =\operatorname{delta}_{s} \operatorname{delta}_{t} \Gamma\left[X_{s}, X_{t}\right] \\
H_{t}^{\#} & =\operatorname{gamma}_{t} X_{t}^{\#} & \Gamma\left[H_{s}, H_{t}\right] & =\text { gamma }_{s} \operatorname{gamma}_{t} \Gamma\left[X_{s}, X_{t}\right] \\
\Gamma\left[H_{t}\right] & =\operatorname{gamma}_{t}^{2} \Gamma\left[X_{t}\right] & \Gamma\left[V_{s}, H_{t}\right] & =\operatorname{delta}_{s} \operatorname{gamma}_{t} \Gamma\left[X_{s}, X_{t}\right] \\
\Gamma\left[X_{t}\right] & =M_{t}^{2} \int_{0}^{t} \frac{X_{u}^{2} \sigma^{2}\left(u, X_{u}\right)}{M_{u}^{2}} d u & \Gamma\left[X_{s}, X_{t}\right] & =M_{s} M_{t} \int_{0}^{s \wedge t} \frac{X_{u}^{2} \sigma^{2}\left(u, X_{u}\right)}{M_{u}^{2}} d u .
\end{aligned}
$$

\section{CONCLUDING REMARKS}

The error calculus based on Dirichlet forms begins at present to be used by modelisators in economics and finance. It is too early to give an account of its applications. What we have done is just presentating how this tool runs through stochastic models including SDE's.

Among the directions of research let us mention that this approach yields new integration by parts formulas which have been shown to be useful to compute the Greeks by Monte Carlo methods. Also, it allows to perform Malliavin calculus on the Monte Carlo sample space, that is after discretization instead of before. Let us indicate briefly the idea :

Consider the error structure

$$
\begin{aligned}
& (\Omega, \mathcal{A}, \mathbb{P}, \mathbb{D}, \Gamma)=((0,1), \mathcal{B}(0,1), d x, \mathrm{~d}, \gamma)^{\mathbf{N}^{*}} \\
& \mathrm{~d} \mathbb{I}=\left\{u \in L^{2}(0,1) \quad x(1-x) u^{\prime}(x) \in H_{0}^{1}(0,1)\right\} \\
& \gamma[u](x)=x^{2}(1-x)^{2} u^{\prime 2}(x) .
\end{aligned}
$$

Let us denote $U_{n}$ the coordinate maps, taking $\mathcal{H}=\ell^{2}$ this structure admits the following gradient : if $F=f\left(U_{1}, U_{2}, \ldots, U_{n}, \ldots\right)$ belongs to $\mathbb{D}$

$$
D F=\left(f_{n}^{\prime}\left(U_{1}, U_{2}, \ldots, U_{n}, \ldots\right) U_{n}\left(1-U_{n}\right)\right)_{n \geq 1}
$$

and if $a \in \ell^{2}$ we have the following integration by parts formula

$$
\mathbb{E}\left[<D F, a>_{\ell^{2}}\right]=-\mathbb{E}\left[F \sum_{n=0}^{\infty} a_{n}\left(1-2 U_{n}\right)\right] .
$$

Let us take for example the following discrete approximation of an SDE:

$$
S_{n+1}=S_{n}+\sigma\left(S_{n}\right)\left(Y_{n+1}-Y_{n}\right), \quad S_{0}=x,
$$

where $Y_{n+1}-Y_{n}=\lambda \xi\left(n+1, U_{n+1}\right)$, we get easily under regularity assumptions on $\xi$ and $\sigma$ :

$$
\begin{aligned}
& \frac{d}{d x} \mathbb{E}\left[\Psi\left(S_{N}\right)\right]=\mathbb{E}\left[\Psi\left(S_{N}\right)\left(\frac{\xi^{\prime \prime}\left(1, U_{1}\right)\left(1+\sigma^{\prime}(x) \xi\left(1, U_{1}\right)\right)}{\sigma(x) \xi^{\prime 2}\left(1, U_{1}\right)}-\frac{\sigma^{\prime}(x)}{\sigma(x)}\right)\right] \\
& \frac{d}{d \lambda} \mathbb{E}\left[\Psi\left(S_{N}\right)\right]=-\mathbb{E}\left[\Psi\left(S_{N}\right) \sum_{n=1}^{N} \frac{d}{d U_{n}} \frac{\xi\left(n, U_{n}\right)}{\xi^{\prime}\left(n, U_{n}\right)}\right] .
\end{aligned}
$$


As a second direction of research, let us sketch how to do a sensitivity analysis of the solution of an SDE with respect to a functional coefficient.

Let us consider that the level dependent volatility of section 5 is a function in a vector space $\Omega_{1}$ equipped with an error structure $\left(\Omega_{1}, \mathcal{A}_{1}, \mathbb{P}_{1}, \mathbb{D}_{1}, \Gamma_{1}\right)$ such that $\mathbb{P}_{1}$-almost every function in $\Omega_{1}$ be of class $\mathcal{C}^{1}$ and Lipschitz, and that the linear form $V_{x}$ defined by

$$
V_{x}(\sigma)=\sigma(x)
$$

belong to $\mathbb{D}_{1}$. Defining the notation $\sigma^{\#}$ by $\sigma^{\#}(x)=\left(V_{x}\right)^{\#}(\sigma)$ we can show that if $Y$ is an erroneous independent random variable defined on an other error structure, the following formula holds

$$
(\sigma(Y))^{\#}=\sigma^{\#}(Y)+\sigma^{\prime}(Y) Y^{\#} .
$$

Then similar computations to those of section 5 can be done. For example suppose $\sigma$ is represented for numerical evaluation as

$$
\sigma(t, x)=\sum_{p, q} a_{p q} t^{p} x^{q}
$$

where the $a_{p q}$ 's are random and erroneous with $\Gamma\left[a_{p q}\right]=a_{p q}^{2}, \quad \Gamma\left[a_{p q}, a_{i j}\right]=0$ if $(p, q) \neq(i, j)$, we obtain that the error on $\sigma$ transfers to $X_{t}$ in the following way:

$$
\Gamma\left[X_{t}\right]=M_{t}^{2} \sum_{p, q}\left(\int_{0}^{t} \frac{s^{p} X_{s}^{q+1}}{M_{s}}\left(d B_{s}-K_{s} d s\right)\right)^{2} a_{p q}^{2}
$$

where $M_{t}, K_{t}$ have the same meaning as in section 5 .

Because estimates of biases are important in financial models especially for pricing, see e.g. Hull and White (1988), let us mention shortly what would be the second order calculus with variances and biases mentionned above in the table 2.1 of section 2. In an error structure $(\Omega, \mathcal{A}, \mathbb{P}, \mathbb{D}, \Gamma)$ the bias of the error on a random variable $X$ (i.e. the conditional expectation of the error) is represented by the generator $A$ of the semi-group canonically associated with the error structure acting on $X$, see Bouleau and Hirsch (1991). It has a domain $\mathcal{D} A$ smaller than $\mathbb{D}$. The functional calculus on $A$ follows the following rules: for all $F \in \mathcal{C}^{2}\left(\mathbb{R}^{d}\right), \forall f_{i}$ locally in $\mathcal{D} A, i=1, \ldots, d$, then $F\left(f_{1}, \ldots, f_{d}\right)$ is locally in $\mathcal{D} A$ and

$$
A[F(f)]=\sum_{i=1}^{d} F_{i}^{\prime}(f) A f_{i}+\frac{1}{2} \sum_{i, j=1}^{d} F_{i j}^{\prime \prime}(f) \Gamma\left[f_{i}, f_{j}\right]
$$

On the Black-Scholes model, with the O-U hypotheses and concerning solely the error due to $\left(B_{t}\right)$, we obtain :

$$
\begin{aligned}
& \Gamma\left[B_{t}\right]=t, \quad \Gamma\left[S_{t}\right]=S_{t}^{2} \sigma^{2} t, \quad A\left[B_{t}\right]=-B_{t}, \\
& A\left[S_{t}\right]=-S_{t} \sigma B_{t}+\frac{1}{2} \sigma^{2} S_{t} t, \quad A\left[V_{t}\right]=\operatorname{delta}_{t} A\left[S_{t}\right]+\frac{1}{2} \text { gamma }_{t} \Gamma\left[S_{t}\right], \\
& A\left[H_{t}\right]=\text { gamma }_{t} A\left[S_{t}\right]+\frac{1}{2} \frac{\partial^{3} F}{\partial x^{3}}\left(t, S_{t}, \sigma, r\right) \Gamma\left[S_{t}\right] .
\end{aligned}
$$

Except at time $t=0$ (since the perturbation 3.3 doesn't move at $t=0$ but we can imagine it starts farther in the past) we see that an error on the path of the stock $\left(S_{t}\right)_{t \geq 0}$ induces biases on the price $V_{t}$ and on the hedge $H_{t}$ (involving the 'Greek' $\left.\frac{\partial^{3} F}{\partial x^{3}}\right)$. May these biases due to a fuzzy stock price be an interpretation of the 
bid-ask ? This interesting question needs certainly more complete investigations since when a transaction occurs the price is erroneous while the amount of stock which is bought or sold is not. Anyhow it is an auspicious project to understand and to take in account the consequences of the bid-ask on pricing and hedging procedures of tractable financial models with the help of the error calculus on variances (operator $\Gamma$ ) and biases (operator $A$ ).

\section{REFERENCES}

Beurling, A., Deny, J. (1958-59): Espaces de Dirichlet, I. le cas élémentaire, Acta Math. 99, 203-224 ; Dirichlet spaces, Proc. Nat. Acad. Sci. U.S.A. 45, 206-215.

Bogachev, V. I., Roeckner, M. (1995): Mehler formula and capacities for infinite dimensional Ornstein-Uhlenbeck processes with general linear drift, Osaka J. Math. 32, 237-274.

Bouleau, N. (1995): Construction of Dirichlet structures, in : Potential theory ICPT 1994, de Gruyter.

Bouleau, N. (2001): Calcul d'erreur complet lipschitzien et formes de Dirichlet, J. Math. pures et appl. 80, 9, 961-976.

Bouleau, N., and Hirsch, F. (1986): Propriétés d'absolue continuité dans les espaces de Dirichlet et application aux EDS, in Sém. probabilité XX, Lecture notes in Mathematics 1204, Springer, 131-161.

Bouleau, N., and Hirsch, F. (1991): Dirichlet forms and analysis on Wiener space, De Gruyter.

Bouleau, N., and Lamberton, D. (1989): Residual risks and hedging strategies in Markovian markets, Stochast. Process. Appl. 33, 131-150.

Dellacherie, and Cl., Meyer, P. A. (1987): Probabilités et potentiel. Hermann.

Feyel, D., and la Pradelle, A. De (1989): Espaces de Sobolev Gaussiens, Ann. Inst. Fourier, 39-4, 875-908.

Fournié, E., Lasry, J.-M., Lebuchoux, J., Lions, P.-L., and Touzi, N. (1999): Application of Malliavin calculus to Monte Carlo methods in finance, Finance and Stochastics, 391-412.

Fukushima, M., Oshima, Y., and Takeda, M. (1994): Dirichlet forms and Markov processes. De Gruyter.

Hull, J. and White, A. (1988) An analysis of the bias in option pricing caused by a stochastic volatility, Advances in Futures and Options Research, vol 3, $29-61$.

LAMBERTon, D., and LAPEYRE, B. (1997): Introduction au calcul stochastic appliqué à la finance. Ellipses.

Ma, Z., and Roeckner, M. ( 1992): Dirichlet forms. Springer.

Malliavin, P. (1997): Stochastic analysis. Springer.

NuAlart, N. (1995): The Malliavin calculus and related topics. Springer. 